\title{
Linkage of $\beta_{1}$-adrenergic stimulation to apoptotic heart cell death through protein kinase $\mathrm{A}$-independent activation of $\mathrm{Ca}^{2+} /$ calmodulin kinase II
}

\author{
Wei-Zhong Zhu, ${ }^{1}$ Shi-Qiang Wang, ${ }^{1}$ Khalid Chakir, ${ }^{1}$ Dongmei Yang, ${ }^{1}$ Tong Zhang, ${ }^{2}$ \\ Joan Heller Brown, ${ }^{2}$ Eric Devic, ${ }^{3}$ Brian K. Kobilka, ${ }^{3}$ Heping Cheng, ${ }^{1}$ and Rui-Ping Xiao ${ }^{1,4}$ \\ ${ }^{1}$ Laboratory of Cardiovascular Science, National Institute on Aging, NIH, Baltimore, Maryland, USA \\ ${ }^{2}$ Department of Pharmacology, University of California, San Diego, La Jolla, California, USA \\ ${ }^{3}$ Department of Medicine and Department of Molecular and Cellular Physiology, Stanford University Medical Center, \\ Stanford, California, USA \\ ${ }^{4}$ Institute of Cardiovascular Sciences, Peking University, Beijing, China
}

$\beta_{1}$-adrenergic receptor $\left(\beta_{1} \mathrm{AR}\right)$ stimulation activates the classic cAMP/protein kinase A (PKA) pathway to regulate vital cellular processes from the change of gene expression to the control of metabolism, muscle contraction, and cell apoptosis. Here we show that sustained $\beta_{1} \mathrm{AR}$ stimulation promotes cardiac myocyte apoptosis by activation of $\mathrm{Ca}^{2+} /$ calmodulin kinase II (CaMKII), independently of PKA signaling. $\beta_{1}$ AR-induced apoptosis is resistant to inhibition of PKA by a specific peptide inhibitor, PKI14-22, or an inactive cAMP analogue, Rp-8-CPT-cAMPS. In contrast, the $\beta_{1} A R$ proapoptotic effect is associated with non-PKA-dependent increases in intracellular $\mathrm{Ca}^{2+}$ and CaMKII activity. Blocking the L-type $\mathrm{Ca}^{2+}$ channel, buffering intracellular $\mathrm{Ca}^{2+}$, or inhibiting CaMKII activity fully protects cardiac myocytes against $\beta_{1} \mathrm{AR}$-induced apoptosis, and overexpressing a cardiac CaMKII isoform, CaMKII- $\delta \mathrm{C}$, markedly exaggerates the $\beta_{1} \mathrm{AR}$ apoptotic effect. These findings indicate that CaMKII constitutes a novel PKA-independent linkage of $\beta_{1}$ AR stimulation to cardiomyocyte apoptosis that has been implicated in the overall process of chronic heart failure.

J. Clin. Invest. 111:617-625 (2003). doi:10.1172/JCI200316326.

\section{Introduction}

Stimulation of $\beta$-adrenergic receptor ( $\beta A R)$, a prototypical $\mathrm{G}$ protein-coupled receptor, is broadly involved in metabolic regulation, growth control, muscle contraction, cell survival, and cell death. In the heart, $\beta A R$ stimulation by catecholamines serves as the most powerful regulatory mechanism to enhance myocardial performance in response to stress or exercise by activating the classic stimulatory pathway comprising the $G$ protein $G_{s}$, adenylyl cyclase, cAMP, and protein kinase A (PKA) $(1,2)$. However, sustained activation of $\beta_{1} \mathrm{AR}$, the predominant $\beta \mathrm{AR}$ subtype expressed in the heart, also induces cardiac myocyte apoptosis (3-6).

Received for publication July 2, 2002, and accepted in revised form December 17, 2002.

Address correspondence to: Rui-Ping Xiao, Laboratory of Cardiovascular Science, Gerontology Research Center, NIA, NIH, 5600 Nathan Shock Drive, Baltimore, Maryland 21224, USA. Phone: (410) 558-8662; Fax: (410) 558-8150;

E-mail: xiaor@grc.nia.nih.gov.

Conflict of interest: The authors have declared that no conflict of interest exists.

Nonstandard abbreviations used: $\beta$-adrenergic receptor $(\beta A R)$; protein kinase A (PKA); $\mathrm{Ca}^{2+} /$ calmodulin-dependent protein kinase II (CaMKII); isoproterenol (ISO); pertussis toxin (PTX); Rp-8-CPT-cAMPS (Rp-cAMP); protein kinase A inhibitor 14-22 (PKI); autocamtide-2-related inhibitory peptide (AIP); sarcoplasmic reticulum (SR); hemagglutinin (HA); phospholamban (PLB).
Apoptotic heart cell death has been implicated in the overall process of myocardial remodeling and the transition from cardiac hypertrophy to chronic heart failure (7-10), an illness afflicting more than five million Americans, with a 5-year mortality greater than $80 \%$ (11). However, the mechanism underlying the $\beta_{1} \mathrm{AR}$ apoptotic effect remains poorly understood.

Previous studies suggested that $\beta_{1}$ AR-induced cardiac myocyte apoptosis was mediated by the cAMP/PKA pathway $(12,13)$, the only known intracellular mechanism underlying $\beta_{1}$ AR-elicited cellular responses $(1,2)$. However, transgenic overexpression of type V (14) or type VI (15) adenylyl cyclase in mouse hearts does not cause cell death, although it markedly augments basal PKA activity (14) and cardiac contractility $(14,15)$. More ironically, in cultured cardiac myocytes or in vivo, selective $\beta_{2}$ AR subtype stimulation elicits a profound cardiac protective effect $(4,5,16)$, in spite of overtly enhanced cAMP formation (16-18).

The goal of the present study is to determine the mechanism of $\beta_{1}$ AR-induced apoptosis. In addition to pharmacological approaches used in WT mouse cardiac myocytes, we created genetically "pure" $\beta_{1}$ AR experimental settings using adult cardiac myocytes from $\beta_{2} A R$ $\mathrm{KO}$ mice (19) or by adenovirus-mediated gene transfer (20) of the mouse $\beta_{1}$ AR in myocytes from $\beta_{1} \beta_{2}$ double knockout (DKO) mice (21). These approaches enabled us to avoid the complicated interactions between the 
coexisting $\beta_{1} \mathrm{AR}$ and $\beta_{2} \mathrm{AR}$ subtypes that exert opposing effects on cardiac cell survival and cell death $(4,5,16)$. In the present study, we demonstrate that $\beta_{1} \mathrm{AR}$-induced cardiac myocyte apoptosis is independent of cAMP and PKA signaling, but requires a novel signaling pathway involving $\mathrm{Ca}^{2+}$ and $\mathrm{Ca}^{2+} /$ calmodulin-dependent protein kinase II (CaMKII).

\section{Methods}

Materials. Isoproterenol (ISO); norepinephrine; prazosin; propranolol; cyclosporin A; FK506; ICI 118,551; $\mathrm{H}-89$; pertussis toxin (PTX); nifedipine; and thapsigargin were purchased from Sigma-Aldrich (St. Louis, Missouri, USA). Rp-8-CPT-cAMPS (Rp-cAMP) was purchased from BIOLOG Life Science Institute (La Jolla, California, USA). PKI14-22 (PKI), autocamtide2-related inhibitory peptide (AIP), KN93, and KN92 were purchased from Calbiochem-Novabiochem Corp. (La Jolla, California, USA).

Cardiac myocyte culture and adenoviral infection. Single cardiac myocytes were enzymatically isolated from the hearts of 2- to 3-month-old WT, $\beta_{2} \mathrm{AR} \mathrm{KO}$, or $\beta_{1} \beta_{2}$ DKO male mice, and then cultured. $\beta_{1} \beta_{2}$ DKO and $\beta_{2} \mathrm{AR}$ KO cells were infected with target gene-carrying adenoviral vectors as described previously (20). Briefly, myocytes were plated at $0.5 \times 10^{4}$ to $1 \times 10^{4}$ cells per $\mathrm{cm}^{2}$ with MEM containing $1.2 \mathrm{mM} \mathrm{Ca}^{2+}$ and $1 \%$ penicillin-streptomycin in culture dishes precoated with 10 $\mu \mathrm{g} / \mathrm{ml}$ mouse laminin. Adenovirus-mediated gene transfer was implemented by adding adenoviral vectors carrying either the mouse $\beta_{1} \mathrm{AR}$ gene (Adv- $\left.\beta_{1} \mathrm{AR}\right)$, the marker gene $\beta$-gal (Adv- $\beta$-gal), or the $\beta$ ARK-ct gene (Adv- $\beta$ ARK-ct, kindly provided by R.J. Lefkowitz and W.J. Koch, Duke University, Durham, North Carolina, USA) to $\beta_{1} \beta_{2}$ DKO cells. Alternatively, hemagglutinintagged (HA-tagged) CaMKII- $\delta$ B vector (Adv-CaMKII$\delta \mathrm{B})$ or HA-tagged CaMKII- $\delta \mathrm{C}$ vector (Adv-CaMKII- $\delta \mathrm{C})$ was added to $\beta_{2} \mathrm{AR}$ KO cells. All transfections were at an MOI of 100 . At the MOI used, almost $100 \%$ of myocytes were positively infected, as evidenced by $\beta$-gal staining (20) or GFP fluorescent signal (Adv-GFP infection, our unpublished data).

After adenoviral infection for 24 hours, culture medium was added with designated reagents, including PKA inhibitors (PKI or Rp-cAMP), CaMKII inhibitors (AIP, KN93, or KN93's inactive analogue, KN92) 1 hour prior to $\beta_{1}$ AR stimulation by ISO. In addition, calcineurin inhibitors (cyclosporin A or FK506), $\beta$ AR antagonists (propranolol or ICI 118,551), or an L-type $\mathrm{Ca}^{2+}$ channel antagonist (nifedipine), or a sarcoplasmic reticulum (SR) $\mathrm{Ca}^{2+}$ pump inhibitor (thapsigargin) were added 1 hour prior to ISO treatment $(1 \mu \mathrm{M})$ in some subsets of experiments. All apoptosis assays were performed after ISO treatment for 24 hours unless otherwise indicated. All dishes were supplemented with ascorbic acid (100 $\mu \mathrm{M}$; Sigma-Aldrich) to prevent ISO oxidation.

TUNEL and Hoechst staining. Nuclear fragmentation was determined in fixed cells (70\% alcohol and 30\% acetone) either by incubating in $10 \mu \mathrm{M}$ Hoechst 33342 or by TUNEL staining with apoptosis detection kits (R\&D Systems Inc., Minneapolis, Minnesota, USA), as previously described (5). The percentage of TUNEL- or Hoechst-positive cells was determined by counting 500-800 cardiac myocytes in 20 randomly chosen fields in each culture dish (with cells pooled from two to three hearts). (In Figure 1, c and d, Figure 2, Figure 4c, Figure $5 b$, and Figure $7 c$, all $n$ values refer to the number of independent experiments, and each data point shows the result from 5,000-6,000 cells, $n=4-8$.) As shown previously (5), the percentage of TUNEL-positive cells is lower than that of Hoechst-positive cells because some apoptotic myocytes are detached and washed away during the TUNEL staining assay.

DNA laddering and cell death ELISA. For DNA laddering, $10 \mu \mathrm{g}$ of DNA was loaded in each lane. The DNA was size-fractionated on a $1.5 \%$ agarose gel in Tris-acetateEDTA buffer and then stained with ethidium bromide (Life Technologies Inc., Carlsbad, California, USA).

For cell death ELISA, myocytes in each group were harvested by trypsinization and then combined with the cells pelleted from media. The cell pellets were washed with PBS and then split into two aliquots: one used for assay of protein abundance to normalize among samples, and the other for extraction of cytoplasmic DNA. DNA was assayed using a commercially available kit to measure nucleosomal DNA (Cell Death Detection ELISA Plus; Roche Molecular Biochemicals, Indianapolis, Indiana, USA). Specifically, $0.20 \mathrm{ml}$ of lysis buffer was used for each aliquot of approximately 100,000 cells. Cells were lysed by gently dispersing the pellet using a pipette tip that was cut back to prevent shearing of cells and release of nuclear DNA. The extraction was performed at room temperature and took 30 minutes. The extract was then centrifuged at low speed and assayed according to the manufacturer's instructions. The control absorbance, measured in myocytes infected by Adv- $\beta_{1} A R$, was considered as $100 \%$ or one unit. Fold increase was obtained by dividing the measured absorbance of an experimental group by the absorbance of the control. All measurements were normalized to the amount of total cellular protein. All measurements were made in triplicate and analysis was performed on at least four independent experiments.

Measurement of intracellular $\mathrm{Ca}^{2+}$. Cultured myocytes in the absence of electrical pacing were loaded with a fluorescent $\mathrm{Ca}^{2+}$ probe, indo-1-acetoxymethyl ester (Molecular Probes Inc., Eugene, Oregon, USA), and were excited at $350 \mathrm{~nm}$. The ratio $(R)$ of fluorescence emission at 410 $\mathrm{nm}$ to that at $490 \mathrm{~nm}$ was used as an index of intracellular $\mathrm{Ca}^{2+}$, as described previously (22). The intracellular free $\mathrm{Ca}^{2+}$ concentration was calculated according to the equation $\left[\mathrm{Ca}^{2+}\right]_{\mathrm{i}}=K_{\mathrm{d}} \beta\left(R-R_{\min }\right) /\left(R_{\max }-R\right)$, where $R_{\min }$ and $R_{\max }$ are the ratio of fluorescence signal at $490 \mathrm{~nm}$ at zero and saturating $\left[\mathrm{Ca}^{2+}\right]_{i}$, respectively; $\beta$ is a constant, and $K_{\mathrm{d}}$ is the dissociation constant of the indicator. In addition, spatial properties of the caffeine-releasable SR $\mathrm{Ca}^{2+}$ store were examined using an LSM410 confocal microscope (Carl Zeiss Jena GmbH, Jena, Germany) with 
UV laser excitation $(351 \mathrm{~nm})$ and dual wavelength ratiometric $(410 / 490 \mathrm{~nm})$ imaging. In another subset of experiments, intracellular $\mathrm{Ca}^{2+}$ transients were measured in cultured myocytes electrically paced at $0.5 \mathrm{~Hz}$.

Phospholamban Ser ${ }^{16}$ phosphorylation, CaMKII autophosphorylation, and CaMKII activity assay. PKA-dependent phosphorylation of phospholamban (PLB) at Ser ${ }^{16}$ was detected by Western blot using a site-specific antibody (Badrilla, West Yorkville, United Kingdom). Total CaMKII protein abundance was assayed by Western blot using an antibody reacting with total CaMKII (Santa Cruz Biotechnology Inc., Santa Cruz, California, USA), whereas autophosphorylated CaMKII was determined with a monoclonal antibody reacting with phosphorylated CaMKII (Affinity BioReagents Inc., Golden, Colorado, USA) (23). CaMKII activity was evaluated with CaMKII assay kits (Upstate Biotechnology Inc., Lake Placid, New York, USA) and a peptide substrate (KKALRRQETVDAL) of the kinase.

Western blot analysis and confocal immunocytochemical imaging of HA-tagged CaMKII- $\delta B$ or CaMKII- $\delta C$. Adenovirus-directed expression of HA-tagged CaMKII- $\delta$ B or CaMKII- $\delta \mathrm{C}$ in $\beta_{2} \mathrm{AR}$ KO cardiomyocytes was examined by Western blot with a monoclonal antibody reacting with HA (Covance Inc., Princeton, New Jersey, USA). To determine subcellular localization of CaMKII- $\delta$ C, cells were incubated overnight at $4{ }^{\circ} \mathrm{C}$ with the $\mathrm{HA}$ monoclonal antibody, followed by an incubation for 4 hours with a Texas Red-conjugated anti-mouse antibody (Vector Laboratories Inc., Burlingame, California,
USA). Immunostaining was then visualized with a scanning confocal microscope (LSM510; Carl Zeiss Jena $\mathrm{GmbH}$ ), as described previously (23).

Statistical analysis. Data were expressed as mean \pm SE. Statistical comparisons used one-way ANOVA followed by the Bonferroni procedure for multiple-group comparisons. $P<0.05$ was considered statistically significant.

\section{Results}

Sustained $\beta_{1} A R$ stimulation delivers a potent apoptotic signal in cardiac myocytes. In $\beta_{1} \beta_{2}$ DKO cells infected with an adenoviral vector encoding $\beta_{1} \mathrm{AR}\left(\mathrm{Adv}-\beta_{1} \mathrm{AR}\right)$ at an MOI of 100 for 24 hours, the $\beta_{1}$ AR density was $550 \pm 46$ $\mathrm{fmol} / \mathrm{mg}$ protein $(n=4)$, which was approximately 16 fold greater than the receptor density in WT cells $(33.5 \pm 1.5 \mathrm{fmol} / \mathrm{mg}$ protein, $n=3)$. Stimulation of the expressed $\beta_{1}$ ARs by ISO $(1 \mu \mathrm{M})$ for 24 hours caused cardiomyocyte death by increased apoptosis (Figure 1a). $\beta_{1} \mathrm{AR}$ stimulation led to a threefold augmentation in cells positive for either TUNEL (Figure 1, a and c) or Hoechst staining (Figure 1d). Apoptotic nuclei appeared blue by TUNEL staining, with a varying degree of chromatin condensation and fragmentation, as indicated by arrows (Figure 1a). Concomitantly, there was a marked increase in DNA fragmentation assayed by cell death ELISA (Figure 1e) and DNA laddering (see Figure 5c) in myocytes subjected to ISO treatment. Propranolol $(10 \mu \mathrm{M})$, a $\beta$ AR antagonist, protected myocytes from ISO-induced apoptosis (Figure 1, a, c, and e, and Figure 5c), indicating that the ISO effect is mediated by

\section{Figure 1}

Inhibition of the CAMP/PKA signaling pathway did not protect myocytes from $\beta_{1}$ ARinduced apoptosis. Cardiac myocytes from $\beta_{1} \beta_{2}$ DKO mice were infected with either Adv$\beta_{1}$ AR or Adv- $\beta$-gal at an MOI of 100. (a) Typical micrographs of TUNEL staining of myocytes. Treatment with $1 \mu \mathrm{M}$ ISO for 24 hours increased the number of apoptotic cells (arrows); the $\beta A R$ antagonist propranolol (10 $\mu \mathrm{M})$, but not the PKA inhibitor PKI $(5 \mu \mathrm{M})$, prevented the $\beta_{1}$ AR apoptotic effect. (b) ISOinduced phosphorylation of PLB at Ser16 (P-PLB-Ser $\left.{ }^{16}\right)$ in the absence $(\mathrm{Ctr})$ or presence of Rp-cAMP $(100 \mu \mathrm{M})$ or PKI $(5 \mu \mathrm{M})$. Similar results were obtained in four other experiments. Pretreatment periods of 1 hour (shown) and 6 hours (not shown) of cells with the PKA inhibitors were equally effective in blocking PKA-dependent PLB phosphorylation in response to ISO treatment $(1 \mu \mathrm{M}$ for 10 minutes). (c-e) Effects of PKA inhibitors on ISO-induced increase in TUNEL staining (c), Hoechst staining (d), or DNA fragmentation as assayed by cell death ELISA (e). Data are presented as mean $\pm \mathrm{SE}(n=4-8$ independent experiments in 5,000-6,000 cells from 10-20 hearts for each group). ${ }^{*} P<0.01$ vs. ISOuntreated cells or those pretreated with propranolol. Prop, propranolol.

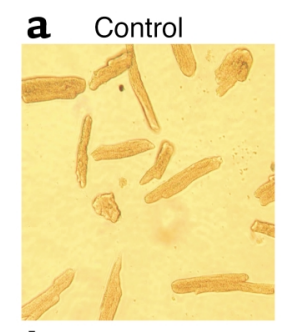

b

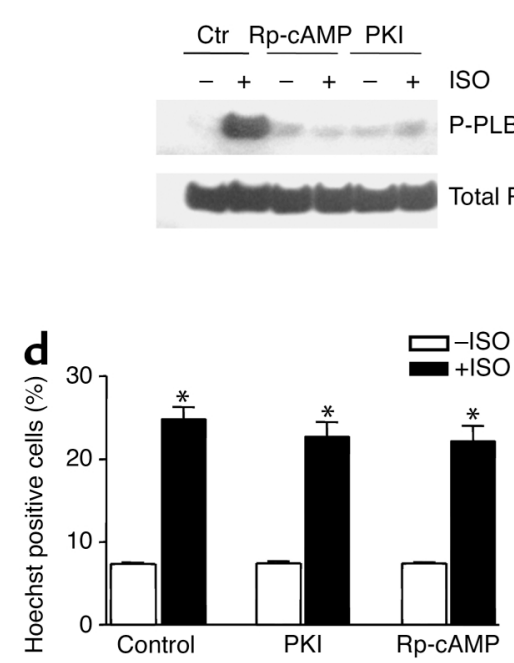

ISO
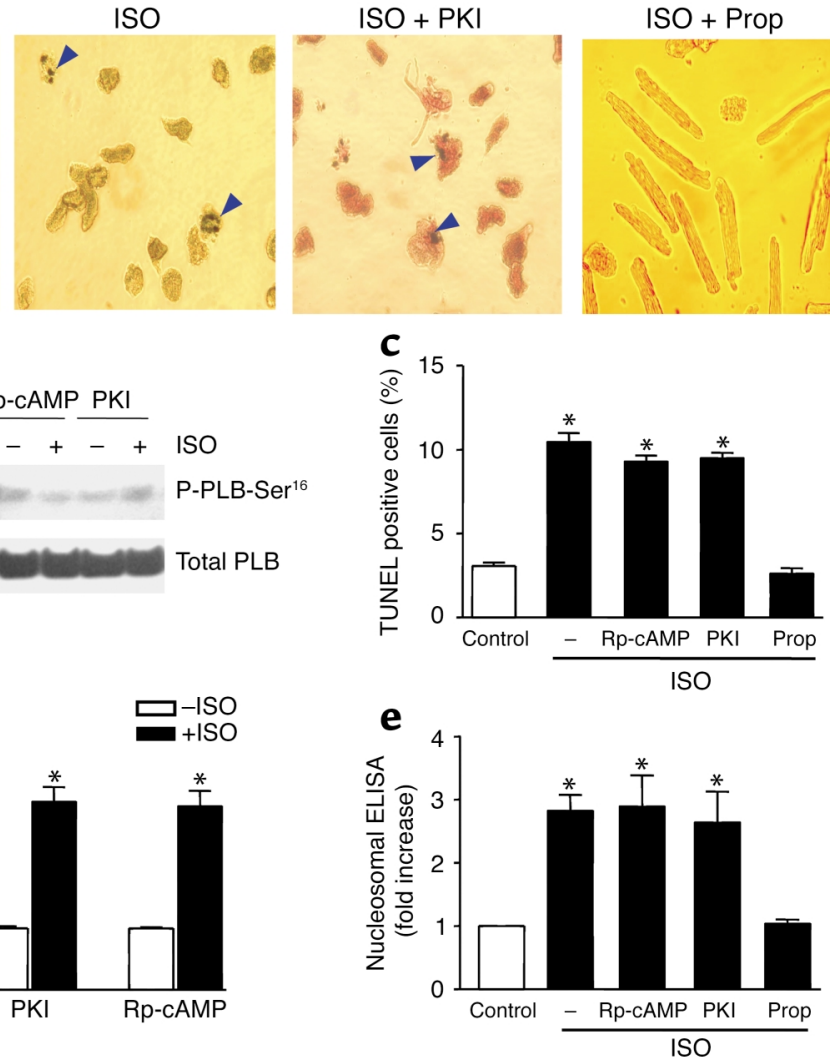

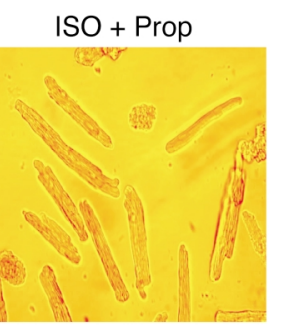

.

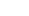

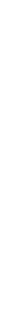

.

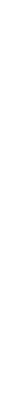



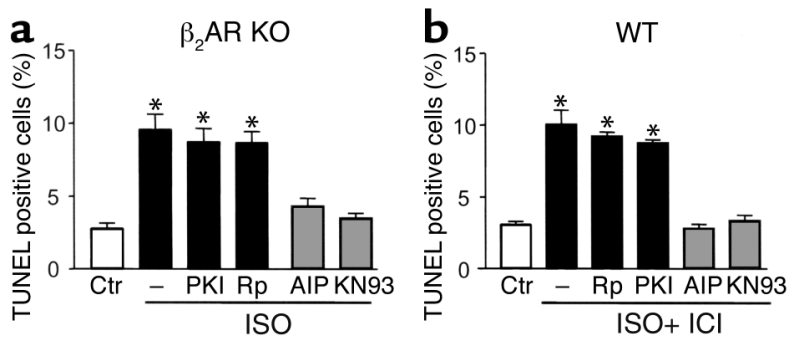

Figure 2

Effects of PKA and CaMKII inhibitors on $\beta_{1}$ AR-mediated increase in TUNEL-positive cells in $\beta_{2}$ AR KO (a) or WT (b) mouse cardiac myocytes. $\beta_{1}$ ARs in $\beta_{2}$ AR KO myocytes were stimulated with $1 \mu \mathrm{M}$ ISO, and $\beta_{1}$ ARs in WT cells were stimulated with $1 \mu \mathrm{M}$ ISO plus the $\beta_{2}$ AR blocker ICI 118,551 $(0.5 \mu \mathrm{M}) .{ }^{*} P<0.01$ vs. ISO-untreated cells or those pretreated with AIP $(10 \mu \mathrm{M})$ or KN93 $(0.5 \mu \mathrm{M})(n=6$ for each group). Rp, Rp-cAMP.

receptor activation. The earliest significant apoptotic effect occurred after 8 hours of ISO treatment, as evidenced by a 2.2 -fold increase in the percentage of TUNEL-positive cells $(n=3, P<0.05)$. Similarly, $\beta_{1} \mathrm{AR}$ stimulation by ISO markedly increased apoptotic cell death in $\beta_{2}$ AR KO myocytes (Figure 2a). Furthermore, selective $\beta_{1}$ AR stimulation by ISO $(1 \mu \mathrm{M})$ in the presence of a $\beta_{2}$ AR blocker, ICI 118,551 $(0.5 \mu \mathrm{M})$, clearly increased WT myocyte apoptosis (Figure $2 \mathrm{~b}$ ). These results validate the relevance of our data in DKO cells expressing $\beta_{1}$ AR using adenoviral gene transfer. Thus, sustained $\beta_{1}$ AR stimulation delivers a potent apoptotic signal, in agreement with previous reports (3-6). Since a similar maximal apoptotic effect occurred in all three experimental systems examined, the receptor density appears not to be a rate-limiting factor in transducing $\beta_{1}$ AR apoptotic signal in cardiac myocytes.

$\beta_{1} A R$-induced apoptosis is independent of cAMP/PKA signaling. To determine the potential role of cAMP/PKA in $\beta_{1}$ AR-mediated myocyte apoptosis, we first used H-89, a widely used PKA inhibitor, and found that $\mathrm{H}-89$ at a high concentration $(20 \mu \mathrm{M})$ protected cardiac myocytes against $\beta_{1} \mathrm{AR}$-induced apoptosis. TUNEL-positive cells were reduced from $11.4 \% \pm 1.1 \%$ to $3.3 \% \pm 0.8 \%$ by $\mathrm{H}-89(n=4, P<0.01)$, consistent with previous reports (12). However, the interpretation of experiments using $\mathrm{H}-89$ is complicated by the recent finding that $\mathrm{H}-89$ is also a potent $\beta$ AR blocker (24). We therefore inhibited this pathway using a highly specific, membrane-permeable peptide PKA inhibitor, PKI (25), and an inactive cAMP analogue, Rp-cAMP (26). Pretreatment of myocytes with either Rp-cAMP $(100 \mu \mathrm{M})$ or PKI $(5 \mu \mathrm{M})$ completely abolished PKA-dependent phosphorylation of PLB (Figure $1 \mathrm{~b}$ ), a key cardiac PKA target protein that regulates the SR $\mathrm{Ca}^{2+}$ pump activity (27), thus validating the effectiveness of these PKA inhibitors in abrogating cAMP/PKA signaling. Surprisingly, neither PKA inhibitor at the same respective concentrations had any significant effect on $\beta_{1} \mathrm{AR}$-mediated apoptosis (Figure 1, a and c-e, and Figure 5c).
The inability of PKA inhibitors to protect cardiac myocytes against $\beta_{1} A R$-induced apoptosis was confirmed in the $\beta_{2} \mathrm{AR} \mathrm{KO}$ model, in which the density and functionality of native $\beta_{1}$ ARs remain unaltered (19), and in WT mouse myocytes subjected to ISO in the presence of $\beta_{2} A R$ blockade. Stimulation of the native $\beta_{1} \mathrm{AR}$ in adult $\beta_{2} \mathrm{AR}$ KO or WT cardiac myocytes by ISO similarly increased TUNEL-positive cells even in the presence of PKI or Rp-cAMP (Figure 2). Thus, while the cAMP/PKA signaling pathway has been thought to be the sole mechanism responsible for $\beta_{1}$-adrenergic responses, $\beta_{1}$ AR apoptotic signal transduction essentially bypasses this pathway.

Role of $G_{\beta \gamma}$ or $G_{i}$ signaling. To identify the molecular mediator for the non-PKA-dependent $\beta_{1}$ AR apoptotic effect, we tested several candidates, including free $G_{\beta \gamma}$ released from heterotrimeric $G_{s}$ proteins or $G$ proteins other than $\mathrm{G}_{s}$ (e.g., $\mathrm{G}_{i}$ proteins). The possible involvement of $G_{\beta \gamma}$ or $G_{i}$ signaling in $\beta_{1}$ AR-mediated apoptosis was examined by adenoviral gene transfer of the C-terminal domain of $\beta A R$ kinase ( $\beta A R K-c t)$ to inhibit $\mathrm{G}_{\beta \gamma}$ signaling (28) and by pretreatment of cells with PTX to disrupt $G_{i}$ signaling (29). Neither $\beta$ ARK-ct nor PTX significantly affected $\beta_{1}$ AR-mediated apoptotic DNA fragmentation (Figure 3). In contrast, both interventions effectively blocked the $\mathrm{G}_{\beta \gamma}$ and $\mathrm{G}_{\mathrm{i}}$-mediated $\beta_{2} \mathrm{AR}$ antiapoptotic effect under similar experimental conditions (5) and prevented $\mathrm{G}_{\beta \gamma}$-mediated activation of PI3K (30). These results rule out the possibility that $G_{\beta \gamma}$ or $G_{i}$ signaling is responsible for $\beta_{1}$ AR-mediated cardiomyocyte apoptosis.

Essential role of $C a^{2+}$ entry and intracellular $\mathrm{Ca}^{2+}$ in $\beta_{1} A R$ apoptotic signaling. It has been shown that altered $\mathrm{Ca}^{2+}$ signaling promotes apoptosis in a variety of cell types (31). We next explored the possibility that $\mathrm{Ca}^{2+}$, instead of cAMP, acts as the second messenger to transmit the $\beta_{1} A R$ apoptotic signal in cardiac myocytes. In Adv$\beta_{1} A R$-infected DKO myocytes, ISO treatment for 3-6 hours (just prior to manifestation of $\beta_{1} \mathrm{AR}$-induced apoptosis) significantly elevated intracellular free $\mathrm{Ca}^{2+}$

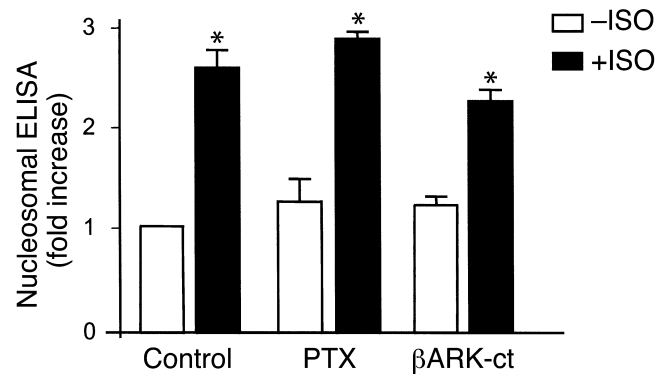

\section{Figure 3}

$\mathrm{G}_{\beta \gamma}$ or $\mathrm{G}_{i}$ signaling is not involved in $\beta_{1}$ AR-induced cardiomyocyte apoptosis. Neither inhibition of $\mathrm{G}_{\beta \gamma}$ signaling by adenoviral expression of $\beta A R K-c t$ nor disruption of $G_{i}$ signaling by pretreatment of cells with PTX $(1 \mu \mathrm{g} / \mathrm{ml}$ for 3 hours $)$ altered ISO-induced $(1 \mu \mathrm{M})$ DNA fragmentation assayed by cell death ELISA in $\beta_{1} \beta_{2}$ DKO cells infected by Adv- $\beta_{1}$ AR. ${ }^{*} P<0.01$ vs. ISO-untreated myocytes. $n=6-7$ independent experiments for each group. 
as measured with the $\mathrm{Ca}^{2+}$-sensitive fluorescent probe indo-1. As shown in Figure $4 \mathrm{a}$, sustained $\beta_{1}$ AR stimulation increased basal cytosolic $\mathrm{Ca}^{2+}$ from $122.8 \pm 5.8(n=32$ cells from six hearts) to $308.7 \pm 9.7 \mathrm{nM}(n=29$ cells from six hearts). This $\mathrm{Ca}^{2+}$ response remained largely intact in the presence of PKI $(5 \mu \mathrm{M})$ but was abolished by nifedipine $(1 \mu \mathrm{M})$, an L-type $\mathrm{Ca}^{2+}$ channel antagonist, indicating an elevation in intracellular $\mathrm{Ca}^{2+}$ mediated by L-type $\mathrm{Ca}^{2+}$ currents $\left(I_{\mathrm{Ca}}\right)$ (Figure $\left.4 \mathrm{a}\right)$. Furthermore, we measured phasic intracellular $\mathrm{Ca}^{2+}$ transients in cultured, paced $(0.5 \mathrm{~Hz})$ cardiac myocytes in the presence and absence of prolonged $\beta_{1}$ AR stimulation by ISO. $\beta_{1} \mathrm{AR}$ stimulation significantly increased both diastolic and systolic $\mathrm{Ca}^{2+}$, with the diastolic effect most prominent, in paced cardiac myocytes (Figure 4b). Thus, $\beta_{1} A R$ stimulation increases cytosolic $\mathrm{Ca}^{2+}$ in both contracting and noncontracting cardiac myocytes. In order to determine whether the altered $\mathrm{Ca}^{2+}$ homeostasis is causally linked to $\beta_{1}$ AR-induced apoptosis, we inhibited $\mathrm{Ca}^{2+}$ entry by nifedipine or buffered intracellular $\mathrm{Ca}^{2+}$ by incubating cells with EGTA-AM. Both treatments rescued cardiac myocytes from $\beta_{1}$ ARinduced apoptosis (Figure 4c).

In addition, we measured $\mathrm{SR} \mathrm{Ca}^{2+}$ load, as indexed by the amplitude of $\mathrm{Ca}^{2+}$ transients generated in response to a bolus administration of caffeine $(20 \mu \mathrm{M})$. We found that sustained $\beta_{1}$ AR stimulation significantly elevated the caffeine-releasable SR Ca ${ }^{2+}$ content, by $38 \%$ (Figure 4, $\mathrm{d}$ and e). Confocal imaging further revealed that the caffeine-induced $\mathrm{Ca}^{2+}$ release was spatially uniform (at the optical resolution), regardless of $\beta_{1} \mathrm{AR}$ stimulation (Figure 4d). Paralyzing SR $\mathrm{Ca}^{2+}$ recycling by inhibiting the SR $\mathrm{Ca}^{2+}$ pump with thapsigargin also protected myocytes from $\beta_{1}$ AR-induced apoptosis (Figure $4 c$ ). Togeth$\mathrm{er}$, these results indicate that the enhanced L-type channel $\mathrm{Ca}^{2+}$ influx, the subsequent increases in cytosolic $\mathrm{Ca}^{2+}$, and the $\mathrm{SR} \mathrm{Ca}^{2+}$ overload are all required for $\beta_{1} \mathrm{AR}$-induced myocyte apoptosis.

$\beta_{1} A R$-induced apoptosis requires PKA-independent activation of CaMKII. To further delineate the specific pathway transducing $\mathrm{Ca}^{2+}$ mediated $\beta_{1} A R$ apoptotic signaling, we examined possible roles of $\mathrm{Ca}^{2+}$-dependent protein phosphatases and kinases, particularly calcineurin and CaMKII. It remains controversial whether activation of calcineurin participates in $\mathrm{Ca}^{2+}$-induced cardiac myocyte apoptosis $(32,33)$, whereas its apoptotic effect is well established in other cell types (34). The present data show that inhibition of calcineurin by cyclosporin A (5 $\mu \mathrm{M})$ or FK506 $(10 \mu \mathrm{M})$ failed to block $\beta_{1}$ ARinduced apoptotic heart cell death, suggesting that calcineurin does not play an essential role in $\beta_{1} \mathrm{AR}$ apoptotic signaling (Figure $5 \mathrm{~b}$ ).

\section{Figure 4}

In sharp contrast, a highly specific membrane-permeable peptide inhibitor of CaMKII, autocamtide-2related inhibitory peptide (AIP, $10 \mu \mathrm{M})(35)$, fully protected myocytes from $\beta_{1} \mathrm{AR}$-induced apoptosis, as evidenced by the typical micrographs (Figure 5a) and the average results of TUNEL staining (Figure $5 \mathrm{~b}$ ). Similar protective effects were observed with another specific CaMKII inhibitor, KN93 $(0.5 \mu \mathrm{M})$, but not its inactive analogue, KN92 (2 $\mu \mathrm{M})$ (Figure 5, a and b). DNA laddering assays confirmed that $\beta_{1}$ AR-induced DNA fragmentation was resistant to the PKA inhibitor PKI, but suppressed by the CaMKII inhibitor KN93 (Figure 5c). Moreover, in either $\beta_{2} \mathrm{AR}$ KO or WT mouse cardiac myocytes, inhibition of CaMKII by AIP or KN93 similarly prevented $\beta_{1}$ AR-mediated apoptosis (Figure 2). Norepinephrine (at $1 \mu \mathrm{M}$ ), a physiological catecholamine, in the presence of an $\alpha_{1}$ AR blocker, prazosin (1 $\mu \mathrm{M})$, similarly promoted apoptosis in WT cells, an effect that was also abolished by KN93 or AIP (data not shown). These results provide the first demonstration
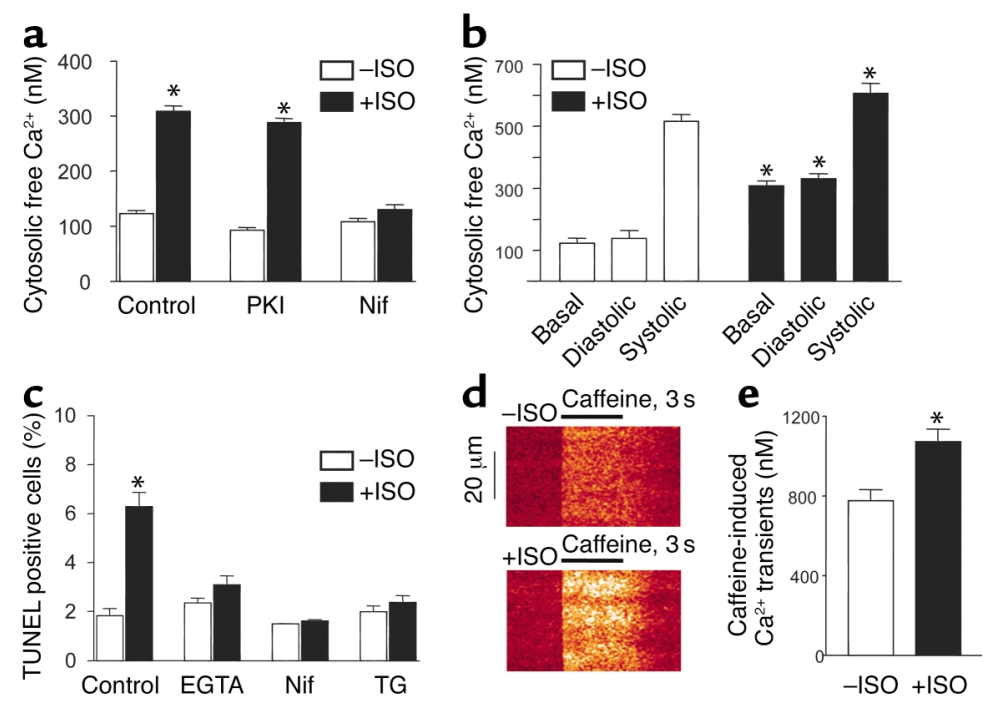

PKA-independent increase in intracellular $\mathrm{Ca}^{2+}$ is essential for the $\beta_{1} \mathrm{AR}$ apoptotic effect. After $\beta_{1} \beta_{2}$ DKO myocytes were infected by Adv- $\beta_{1} A R$, cells were incubated with designated reagents for 1 hour, then ISO $(1 \mu \mathrm{M})$ was added and cells were incubated for another 3-6 hours (a, b, d, and e) or 24 hours (c). (a) Prolonged $\beta_{1} \mathrm{AR}$ stimulation elevated basal intracellular free $\mathrm{Ca}^{2+}$ in unpaced cardiac myocytes. This effect was blocked by the L-type $\mathrm{Ca}^{2+}$ channel antagonist nifedipine $(1 \mu \mathrm{M})$, but not the PKA inhibitor PKI $(5 \mu \mathrm{M}) .{ }^{*} P<0.01$ vs. ISO-untreated groups and those pretreated by nifedipine ( $n=20-35$ cells from six hearts). (b) Intracellular $\mathrm{Ca}^{2+}$ transients were measured in a subset of cells electrically paced at $0.5 \mathrm{~Hz}$ for at least 10 minutes in the absence $(n=29$ cells from four hearts) and presence ( $n=22$ cells from four hearts) of sustained $\beta_{1}$ AR stimulation by ISO. ${ }^{*} P<0.05$ vs. ISO-untreated myocytes. (c) Effects of nifedipine, EGTA-AM ( $\left.1 \mu M\right)$, or the SR ATPase inhibitor thapsigargin ( $1 \mu \mathrm{M})$ on $\beta_{1} A R$-induced increase in TUNEL-positive cells. ${ }^{*} P<0.01$ vs. ISO-untreated myocytes and those pretreated with EGTA-AM, nifedipine, or thapsigargin $(n=4-8)$. (d) Representative confocal linescan images of caffeine-elicited SR Ca ${ }^{2+}$ release in ISO-treated $(1 \mu \mathrm{M}, 3$ hours, bottom) and untreated cells (top). The $x$ axis shows the time courses for caffeine treatment, and the $y$ axis shows the spatial profiles of $\mathrm{Ca}^{2+}$ transients along a scan line inside the cell. (e) Average amplitude of caffeine-elicited $\mathrm{Ca}^{2+}$ transients in ISOtreated or untreated group. ${ }^{*} P<0.01 \mathrm{vs}$. ISO-untreated myocytes. $n=25-30$ cells from six hearts in each group. Nif, nifedipine; TG, thapsigargin. 


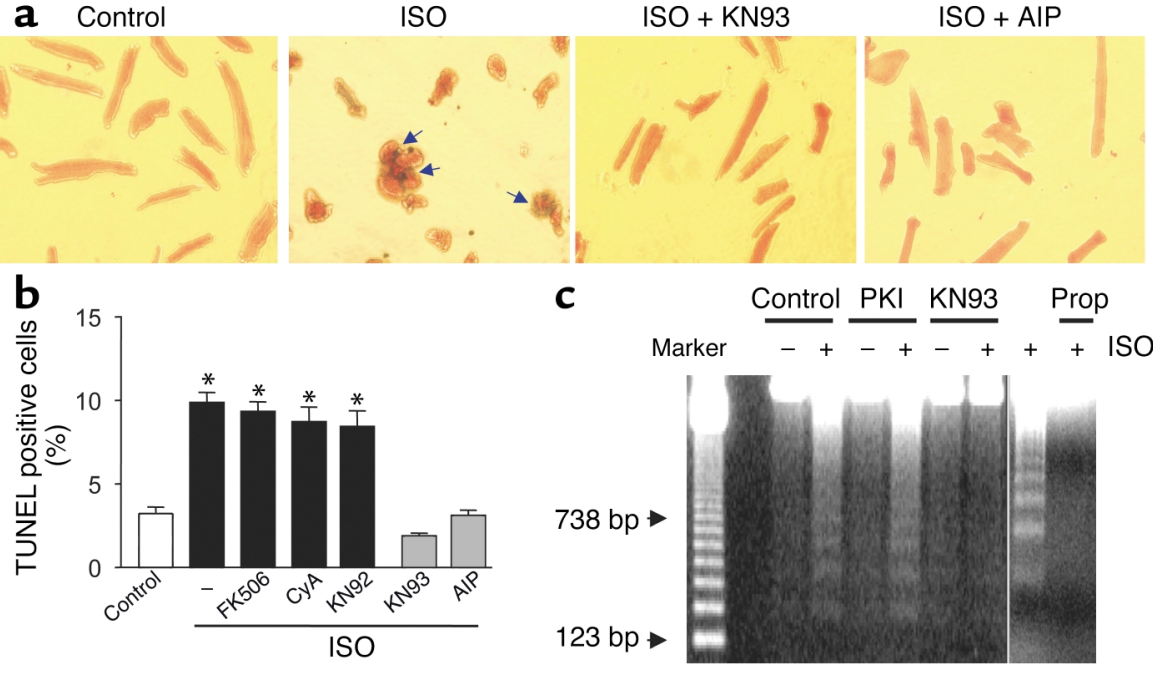

\section{Figure 5}

Role of CaMKII and calcineurin in the $\beta_{1}$ AR-mediated apoptotic effect. After $\beta_{1} \beta_{2}$ DKO myocytes were infected by Adv- $\beta_{1} A R$, cells were pretreated with the CaMKII inhibitors AIP $(10 \mu \mathrm{M})$ or KN93 $(0.5 \mu \mathrm{M})$ or the inactive KN93 analogue KN92 $(2 \mu \mathrm{M})$; with the calcineurin inhibitors FK506 (10 $\mu \mathrm{M})$ or cyclosporin $\mathrm{A}(5 \mu \mathrm{M})$; with the PKA inhibitor PKI $(5 \mu \mathrm{M})$; or with the $\mathrm{Ca}^{2+}$ channel blocker nifedipine $(1 \mu \mathrm{M})$, all for 1 hour (except 3 hours for AIP) prior to administration of $1 \mu \mathrm{M}$ ISO. Apoptosis was assessed after incubation for another 24 hours. (a) AIP or KN93 fully protected cardiomyocytes against ISO-elicited apoptosis. Arrows indicate TUNEL-positive nuclei. (b) The ISO-induced increase in TUNEL-positive cells was prevented by AIP or KN93, but not the inactive KN93 analogue KN92 or the calcineurin inhibitors FK506 or cyclosporin A. $n=4-8 .{ }^{*} P<0.01$ versus ISO-untreated groups or those treated with KN93 or AIP. (c) ISO-induced DNA laddering in the absence (control) or presence of KN93, PKI, or the $\beta A R$ antagonist propranolol. Similar results were obtained in four other experiments. CyA, cyclosporin A.

that activation of CaMKII, rather than PKA, is required for $\beta_{1}$ AR-induced apoptosis in the heart.

The pivotal role of CaMKII in $\beta_{1}$ AR-mediated apoptotic signaling was reinforced by data on the pharmacological profile of $\beta_{1} \mathrm{AR}$-induced CaMKII activation. In Adv- $\beta_{1} A R$-infected DKO cells, ISO stimulation for 6 hours increased the level of autophosphorylated CaMKII, an active form of the kinase (36), without altering the abundance of the kinase protein (Figure 6a). Notably, the increase in CaMKII activity was PKIresistant, but was abolished by KN93 and AIP (Figure $6, a-c)$ at the same respective concentrations that blocked the $\beta_{1}$ AR-mediated apoptotic effect. Since CaMKII activity was elevated prior to the manifestation of cardiomyocyte apoptosis, we conclude that CaMKII activation constitutes an early $\beta_{1}$ AR-elicited event to signal cell apoptosis.

Overexpression of CaMKII- $\delta$ C exaggerates $\beta_{1} A R$-induced apoptosis. Based on the aforementioned results, an increase in CaMKII abundance should enhance the $\beta_{1}$ AR apoptotic effect in cardiac myocytes. To test this hypothesis, we expressed HA-tagged CaMKII- $\delta \mathrm{C}$, a predominant cardiac cytoplasmic CaMKII isoform (37), or CaMKII- $\delta \mathrm{B}$, a nuclear CaMKII isoform (37), in $\beta_{2} \mathrm{AR}$ $\mathrm{KO}$ myocytes. Confocal immunocytochemical analysis confirmed that the expressed CaMKII- $\delta$ C was distributed in cytoplasm and the surface membranes, including the transverse tubules (Figure 7a, II and III), but not in the nuclei (Figure $7 \mathrm{a}, \mathrm{III})$, whereas CaMKII- $\delta \mathrm{B}$ was concentrated in the nuclei (Figure 7a, IV). Expression of HA-tagged CaMKII$\delta C$ was also confirmed by Western blotting using a monoclonal antibody reacting with HA (Figure 7b). Remarkably, overexpression of CaMKII- $\delta C$ shifted the dose-response curve of ISOinduced apoptosis leftward by nearly an order of magnitude $\left(\mathrm{EC}_{50}, 1.1 \mathrm{nM}\right.$ and 9.0 $\mathrm{nM}$ for myocytes infected by $A d v-C a M K I I-\delta C$ and $A d v-\beta-$ gal, respectively) and increased the maximal apoptotic effect by $50 \%$, whereas it exerted no appreciable apoptotic effect in the absence of $\beta_{1} \mathrm{AR}$ stimulation by ISO (Figure 7c). Again, inhibition of CaMKII by KN93 in cardiac myocytes overexpressing CaMKII- $\delta C$ abolished the $\beta_{1}$ AR apoptotic effect over a wide range of agonist concentrations (Figure 7c). By contrast, overexpression of CaMKII- $\delta \mathrm{B}$ neither enhanced nor reduced $\beta_{1}$ ARinduced myocyte apoptosis (data not shown), indicating that CaMKII- $\delta \mathrm{C}$ but not CaMKII- $\delta \mathrm{B}$ is involved in $\beta_{1}$ AR apoptotic signaling. The fact that increased CaMKII- $\delta$ C abundance exaggerates the $\beta_{1}$ AR apoptotic effect without altering basal cell apoptosis substantiates the conclusion that CaMKII mediates the $\beta_{1} \mathrm{AR}$ apoptotic signal.

\section{Discussion}

$\beta_{1}$ AR apoptotic signaling pathway. The prevalent theory of $\beta_{1} \mathrm{AR}$ signal transduction is that the cAMP/PKA pathway is solely responsible for $\beta_{1}$ AR-mediated cellular responses. A close inspection of studies to date, however, reveals no convincing evidence to validate that this is also the case for $\beta_{1}$ AR-evoked apoptotic signal in the heart. Using two genetically defined $\beta_{1} A R$ systems, we have provided the first documentation that sustained $\beta_{1}$ AR stimulation delivers a powerful cardiac apoptotic signal via a CaMKII-dependent, rather than a PKAdependent, mechanism. This conclusion is based on several lines of evidence. First, inhibition of PKA by a specific peptide inhibitor, $\mathrm{PKI}$, or an inactive cAMP analogue, Rp-cAMP, does not affect $\beta_{1}$ AR-induced myocyte apoptosis under conditions in which PKA-mediated protein phosphorylation is completely blocked. Second, sustained $\beta_{1} A R$ stimulation elicits PKA-independent augmentation of intracellular $\mathrm{Ca}^{2+}$ as well as $\mathrm{SR}^{2} a^{2+}$ 

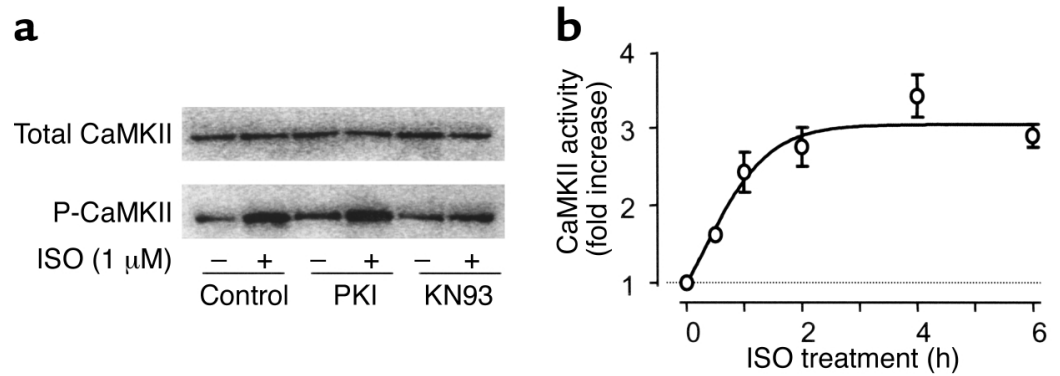

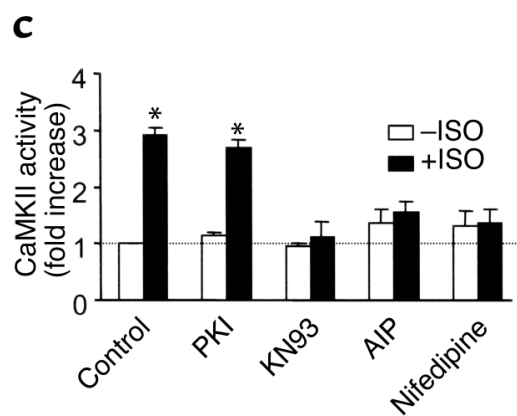

\section{Figure 6}

Temporal and pharmacological profiles of CaMKII activation in response to $\beta_{1}$ AR stimulation. (a) In Adv- $\beta_{1} A R$-infected $\beta_{1} \beta_{2}$ DKO myocytes, $\beta_{1}$ AR stimulation ( $1 \mu \mathrm{M}$ ISO for 6 hours) increased CaMKII autophosphorylation. This effect was blocked by KN93 (5 $\mu$ M) but not by the PKA inhibitor PKI $(5 \mu \mathrm{M})$. Similar results were obtained in three other experiments. (b) Time course of ISO-induced increase in CaMKII activity assayed by ${ }^{32} \mathrm{P}$ incorporation into a specific peptide substrate of the kinase (see Methods; $n=4$ for each data point). (c) Pharmacological profile of CaMKII activation $(n=4-6) .{ }^{*} P<0.01 \mathrm{vs}$. cells in the absence of ISO or those in the presence of KN93, AIP, or nifedipine. P-CaMKII, autophosphorylated CaMKII.

load in both resting and electrically paced myocytes, and CaMKII is activated in a time-dependent fashion. More importantly, either blocking $I_{\mathrm{Ca}}$, buffering intracellular $\mathrm{Ca}^{2+}$, or inhibiting CaMKII activity fully protects cardiac myocytes against $\beta_{1}$ AR-induced apoptosis. The essential role of CaMKII in $\beta_{1} \mathrm{AR}$ apoptotic signaling is also corroborated by the fact that overexpression of a cardiac isoform of CaMKII, CaMKII- $\delta$ C, markedly enhances $\beta_{1}$ AR-induced apoptosis. Similarly, in cultured adult rat cardiac myocytes, $\beta_{1}$ AR-mediated apoptosis is blocked by nifedipine or CaMKII inhibition with KN93, but not by PKA inhibition with PKI (data not shown). Thus, we conclude that the $\beta_{1}$ AR-evoked apoptotic signal is delivered by a PKA-independent, CaMKII-mediated signaling pathway. Nevertheless, this should not be taken as evidence that basal CaMKII activation (such as in the beating heart) alone is sufficient to induce apoptosis. Our previous studies have shown that $2.0-\mathrm{Hz}$ electrical pacing is able to augment CaMKII activation, as shown by a twofold increase in CaMKII-dependent phosphorylation of PLB at $\mathrm{Thr}^{17}$, and that the effect of pacing on PLB-Thr ${ }^{17}$ phosphorylation is synergistically enhanced to sixfold when combined with $\beta_{1}$ AR stimulation in freshly isolated rat cardiac myocytes (38), indicating that $\beta_{1}$ AR stimulation and electrical pacing exert a synergistic effect on CaMKII activation in cardiac myocytes. Although unexpected, the present finding that the $\beta_{1} \mathrm{AR}$ apoptotic effect is PKA-independent is supported by previous observations that transgenic overexpression of adenylyl cyclase and the resultant elevation of intracellular cAMP in mouse hearts are dissociated from myocyte apoptosis $(14,15)$, and that $\beta_{2} \mathrm{AR}$ stimulation exhibits a profound antiapoptotic effect despite elevated intracellular cAMP formation in cardiac myocytes $(3-5,16)$. Together, the present and previous studies indicate that an increase in cAMP/PKA signaling does not necessarily cause heart cell apoptosis. This perhaps reflects distinct compartmentation of intracellular cAMP under different circumstances $(2,26,39-42)$. In

contrast, the linkage of sustained $\beta_{1}$ AR stimulation to cardiac myocyte apoptosis by the multifunctional protein kinase, CaMKII, is in general agreement with recent findings that, in naive cell lines, numerous insulting factors including UV light, TNF- $\alpha$ (43), and protein phosphatase inhibitors (44) can activate CaMKII, which then contributes to the insult-induced apoptosis.

Distinct signaling modes underlie sustained versus acute $\beta_{1}$ AR stimulation. Given that both $\mathrm{Ca}^{2+}$ and cAMP serve as the second messenger, $\beta_{1}$ AR signal transduction bifurcates into two pathways mediated by CaMKII and PKA, respectively. Interestingly, the two pathways are called upon in tandem to fulfill distinctly different functional roles. Acute $\beta_{1}$ AR stimulation rapidly

a

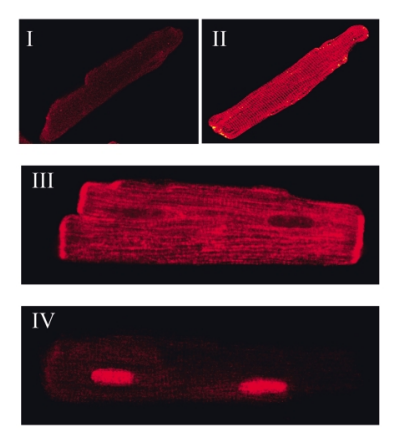

Figure 7

Overexpression of CaMKII- $\delta C$ exaggerates $\beta_{1} A R$-induced myocyte apoptosis. (a) Confocal imaging of HA immunofluorescence in typical $\beta_{2} A R$ KO myocytes expressing either $\beta$-gal (I), or HA-tagged CaMKII- $\delta C$ (II, cell surface scan; III, cell nucleus level scan), or HA-tagged CaMKII- $\delta$ B (IV). (b) Expression of HA-tagged CaMKII- $\delta C$ assayed by Western blot with an antibody reacting with $H A$. (c) Dose responses of $\beta_{1} A R$-induced increase in apoptotic cells in $\beta_{2} A R K O$ myocytes infected by Adv-CaMKII- $\delta C$ (with or without KN93) or Adv- $\beta$-gal ( $n=6$ for each data point). 
activates the cAMP/PKA pathway, with the peak response within 1 minute (45), whereas prolonged $\beta_{1}$ AR stimulation causes desensitization of cAMP/ PKA signaling within 30 minutes $(1,24)$. This fast cAMP/PKA response is crucial to sympathetic control over the heart rate and myocardial contraction, allowing the heart to increase its output within seconds in response to a "fight-or-flight" situation. In contrast, the newly identified CaMKII signaling is evoked gradually and appears to contribute little to acute $\beta_{1} \mathrm{AR}$ contractile response, but is essential to the $\beta_{1} \mathrm{AR}$ cardiac apoptotic effect.

The slow kinetics of CaMKII activation may reflect a cumulative increase in intracellular $\mathrm{Ca}^{2+}$ due to a small but persistent $\mathrm{Ca}^{2+}$ entry through L-type channels. This interpretation is consistent with the facts that CaMKII activation is blocked by the L-type channel antagonist niphedipine and that CaMKII- $\delta \mathrm{C}$ is enriched beneath cell surface membranes, thus permitting an intimate interplay between CaMKII and the L-type channel (23). Alternatively, the gradual elevation of CaMKII activity may reflect that multiple steps, e.g., upregulation of intermediate signaling components, are involved in transducing receptor signal to the kinase activation.

Regardless of the exact mechanism, the time-dependent switch of signaling modes during enduring receptor stimulation may represent a new paradigm of $G$ protein-coupled receptor signal transduction. In this regard, it has recently been shown that $\beta_{2}$ AR stimulation induces a switch from cAMP/PKA signaling to a $\mathrm{G}_{\mathrm{i}}$-dependent MAPK signaling pathway (46), and that these two events are causally linked, i.e., activation of cAMP/PKA is a prerequisite for the receptor coupling to $G_{i}$ proteins and the subsequent activation of the MAPK pathway (46). In the case for sustained $\beta_{1} A R$ stimulation, however, the gradual activation of CaMKII is independent of PKA signaling (Figure 6). Both examples show a time-dependent homologous regulation of $G$ protein-coupled receptor signaling.

Regarding possible mechanisms underlying the non-PKA-dependent increase in intracellular $\mathrm{Ca}^{2+}$, it has been proposed that persistent $\beta_{1} \mathrm{AR}$ stimulation increases $I_{\mathrm{Ca}}$ via a direct coupling of $\mathrm{G}_{\mathrm{s} \alpha}$ to the $\mathrm{Ca}^{2+}$ channel (47). More recently, it has been shown that cardiac-specific overexpression of $\mathrm{G}_{\mathrm{s \alpha}}$ similarly augments $I_{\mathrm{Ca}}$ amplitude in a PKA-independent manner (48). Both lines of evidence support the idea that PKA-independent cross-talk between $\mathrm{G}_{s \alpha}$ and the L-type $\mathrm{Ca}^{2+}$ channel may contribute to $\beta_{1} \mathrm{AR}$-induced increase in intracellular $\mathrm{Ca}^{2+}$ and subsequent activation of CaMKII.

Pathophysiological relevance of $\beta_{1} A R$ apoptotic signaling. Increasing evidence indicates that prolonged $\beta_{1} A R$ stimulation exerts a cardiotoxic effect that often outweighs the short-term gain in cardiac contractile support. Both in vivo and in vitro studies have shown that enhanced $\beta_{1} \mathrm{AR}$ signaling by either selective receptor stimulation or receptor overexpression increases proapoptotic protein levels and promotes cardiac apoptosis (3-6). Since cardiac myocytes are terminally differentiated cells, preventing the loss of irreplaceable cells is critical for the maintenance of normal cardiac function. These studies explain, at least in part, the poor prognosis of heart failure patients with tonically elevated plasma norepinephrine, an endogenous $\beta_{1} A R$ agonist (49), as well as the beneficial effects of $\beta A R$ blockers, particularly $\beta_{1}$ AR blockers in chronic heart failure (50). In light of the present findings, an increase in intracellular $\mathrm{Ca}^{2+}$ and subsequent activation of CaMKII, rather than the long-suspected cAMP/PKA cascade, are particularly cardiotoxic in terms of cardiac myocyte apoptosis. Thus, unraveling the new pathway and the novel signaling mode of $\beta_{1}$ AR signal transduction hold promise for identifying key therapeutic targets and new strategies for minimizing detrimental effects of $\beta_{1}$ AR stimulation in the failing heart.

\section{Acknowledgments}

We thank E.G. Lakatta, M. Crow, M. Mattson, A. Chelsey, N.M. Soldatov, and D.R. Abernethy for valuable discussions, and B. Ziman for excellent technical support. This work was supported by the NIH Intramural Research Program (K. Chakir, H. Cheng, and R.-P. Xiao) and by Major State Basic Research Development Program (grant no. 973) of China (R.-P. Xiao).

1. Dohlman, H.G., Thorner, J., Caron, M.G., and Lefkowitz, R.J. 1991. Model systems for the study of seven-transmembrane-segment receptors. Annu. Rev. Biochem. 60:653-688.

2. Xiao, R.P. 2001. $\beta$-adrenergic signaling in the heart: dual coupling of the $\beta_{2}$-adrenergic receptor to $G_{s}$ and $G_{i}$ proteins. Sci. STKE. 104:RE15.

3. Zaugg, M., et al. 2000. $\beta$-adrenergic receptor subtypes differentially affect apoptosis in adult rat ventricular myocytes. Circulation. 102:344-350.

4. Communal, C., Singh, K., Sawyer, D.B., and Colucci, W.S. 1999. Opposing effects of $\beta_{1^{-}}$and $\beta_{2}$-adrenergic receptors on cardiac myocyte apoptosis: role of a pertussis toxin-sensitive $G$ protein. Circulation. 100:2210-2212.

5. Zhu, W.Z., et al. 2001. Dual modulation of cell survival and cell death by $\beta_{2}$-adrenergic signaling in adult mouse cardiac myocytes. Proc. Natl. Acad. Sci. U. S. A. 98:1607-1612.

6. Bisognano, J.D., et al. 2000. Myocardial-directed overexpression of the human $\beta_{1}$-adrenergic receptor in transgenic mice. J. Mol. Cell. Cardiol. 32:817-830.

7. Kang, P.M., and Izumo, S. 2000. Apoptosis and heart failure: a critical review of the literature. Circ. Res. 86:1107-1113.

8. Narula, J., et al. 1996. Apoptosis in myocytes in end-stage heart failure. N. Engl. J. Med. 335:1182-1189.

9. Olivetti, G., et al. 1997. Apoptosis in the failing human heart. N. Engl. J. Med. 336:1131-1341.

10. Adams, J.W., et al. 1998. Enhanced $\mathrm{G}_{\alpha q}$ signaling: a common pathway mediates cardiac hypertrophy and apoptotic heart failure. Proc. Natl. Acad. Sci. U. S. A. 95:10140-10145.

11. Konstam, M.A., and Remme, W.J. 1998. Treatment guidelines in heart failure. Prog. Cardiovasc. Dis. 41:65-72.

12. Communal, C., Singh, K., Pimentel, D.R., and Colucci, W.S. 1998. Norepinephrine stimulates apoptosis in adult rat ventricular myocytes by activation of the $\beta$-adrenergic pathway. Circulation. 98:1329-1334.

13. Iwai-Kanai, E., et al. 1999. $\alpha$ - and $\beta$-adrenergic pathways differentially regulate cell type-specific apoptosis in rat cardiac myocytes. Circulation. 100:305-311.

14. Tepe, N.M., et al. 1999. Altering the receptor-effector ratio by transgenic overexpression of type $\mathrm{V}$ adenylyl cyclase: enhanced basal catalytic activity and function without increased cardiomyocyte $\beta$-adrenergic signaling. Biochemistry. 38:16706-16713.

15. Gao, M.H., et al. 1999. Adenylyl cyclase increases responsiveness to catecholamine stimulation in transgenic mice. Circulation. 99:1618-1622.

16. Chesley, A., et al. 2000 . The $\beta_{2}$-adrenergic receptor delivers an antiapoptotic signal to cardiac myocytes through $\mathrm{G}_{\mathrm{i}}$-dependent coupling to phosphatidylinositol 3'-kinase. Circ. Res. 87:1172-1179.

17. Milano, C.A., et al. 1994. Enhanced myocardial function in transgenic mice overexpressing the $\beta_{2}$-adrenergic receptor. Science. 264:582-586.

18. Zhou, Y.Y., et al. 2000. Spontaneous activation of $\beta_{2}$ - but not $\beta_{1}$-adreno- 
ceptors expressed in cardiac myocytes from $\beta_{1} \beta_{2}$ double knockout mice. Mol. Pharmacol. 58:887-894.

19. Chruscinski, A.J., et al. 1999. Targeted disruption of the $\beta_{2}$-adrenergic receptor gene. J. Biol. Chem. 274:16694-16700.

20. Zhou, Y.Y., et al. 2000. Culture and adenoviral infection of adult mouse cardiac myocytes: methods for cellular genetic physiology. Am. J. Physiol. 279:H429-H436.

21. Rohrer, D.K., Chruscinski, A., Schauble, E.H., Bernstein, D., and Kobilka, B.K. 1999. Cardiovascular and metabolic alterations in mice lacking both $\beta_{1}$ - and $\beta_{2}$-adrenergic receptors. J. Biol. Chem. 274:16701-16708.

22. Spurgeon, H.A., et al. 1990. Simultaneous measurement of $\mathrm{Ca}^{2+}$, contraction, and potential in cardiac myocytes. Am. J. Physiol. 258:H574-H586.

23. Xiao, R.P., Cheng, H., Lederer, W.J., Suzuki, T., and Lakatta, E.G. 1994. Dual regulation of $\mathrm{Ca}^{2+} /$ calmodulin-dependent kinase II activity by membrane voltage and by calcium influx. Proc. Natl. Acad. Sci. U. S. A. 91:9659-9663.

24. Penn, R.B., Parent, J.L., Pronin, A.N., Panettieri, R.A., Jr., and Benovic, J. 1999. Pharmacological inhibition of protein kinases in intact cells: antagonism of $\beta$-adrenergic receptor ligand binding by $\mathrm{H}-89$ reveals limitations of usefulness. J. Pharmacol. Exp. Ther. 288:428-437.

25 . Zheng, M., et al. 2000. $\beta_{2}$-adrenergic receptor-induced p38 MAPK activation is mediated by protein kinase $A$ rather than by $G_{i}$ or $G_{\beta \gamma}$ in adult mouse cardiomyocytes. J. Biol. Chem. 275:40635-40640.

26. Zhou, Y.Y., et al. 1997. Localized cAMP-dependent signaling mediates $\beta_{2}$-adrenergic modulation of cardiac excitation-contraction coupling. Am. J. Physiol. 273:H1611-H1618.

27. Koss, K.L., and Kranias, E.G. 1996. Phospholamban: a prominent regulator of myocardial contractility. Circ. Res. 79:1059-1063.

28. Koch, W.J., Hawes, B.E., Inglese, J., Luttrell, L.M., and Lefkowitz, R.J 1994. Cellular expression of the carboxyl terminus of a $G$ protein-coupled receptor kinase attenuates $\mathrm{G}_{\beta \gamma}$-mediated signaling. J. Biol. Chem. 269:6193-6197.

29. Xiao, R.P., et al. 1999. Coupling of $\beta_{2}$-adrenoceptor to $G_{i}$ proteins and its physiological relevance in murine cardiac myocytes. Circ. Res. 84:43-52.

30. Jo, S.H., Leblais, V., Wang, P.H., Crow, M.T., and Xiao, R.P. 2002. Phosphatidylinositol 3-kinase functionally compartmentalizes the concurrent $\mathrm{G}_{\mathrm{s}}$ signaling during $\beta_{2}$-adrenergic stimulation. Circ. Res. 91:46-53.

31. Berridge, M.J., Bootman, M.D., and Lipp, P. 1998. Calcium-a life and death signal. Nature. 395:645-648.

32. Saito, S., et al. 2000. $\beta$-Adrenergic pathway induces apoptosis through calcineurin activation in cardiac myocytes. J. Biol. Chem. 275:34528-34533.

33. Kakita, T., et al. 2001. Calcineurin pathway is required for endothelin-1mediated protection against oxidant stress-induced apoptosis in cardiac myocytes. Circ. Res. 88:1239-1246.

34. Wang, H.G., et al. 1999. $\mathrm{Ca}^{2+}$-induced apoptosis through calcineurin dephosphorylation of BAD. Science. 284:339-343.
35. Vinogradova, T.M., et al. 2000. Sinoatrial node pacemaker activity requires $\mathrm{Ca}^{2+} /$ calmodulin-dependent protein kinase II activation. Circ. Res. 87:760-767.

36. Braun, A.P., and Schulman, H. 1995. The multifunctional calcium/calmodulin-dependent protein kinase: from form to function. Annu. Rev. Physiol. 57:417-445.

37. Edman, C.F., and Schulman, H. 1994. Identification and characterization of $\delta \mathrm{B}-\mathrm{CaM}$ kinase and $\delta \mathrm{C}-\mathrm{CaM}$ kinase from rat heart, two new multifunctional $\mathrm{Ca}^{2+} /$ calmodulin-dependent protein kinase isoforms. Biochim. Biophys. Acta. 1221:89-101.

38. Hagemann, D., et al. 2000. Frequency-encoding Thr $^{17}$ phospholamban phosphorylation is independent of $\operatorname{Ser}^{16}$ phosphorylation in cardiac myocytes. J. Biol. Chem. 275:22532-22536.

39. Zaccolo, M., and Pozzan, T. 2002. Discrete microdomains with high concentration of cAMP in stimulated rat neonatal cardiac myocytes. Science. 295:1711-1715.

40. Ostrom, R.S., et al. 2001. Receptor number and caveolar co-localization determine receptor coupling efficiency to adenylyl cyclase. J. Biol. Chem. 276:42063-42069.

41. Rybin, V.O., Xu, X., Lisanti, M.P., and Steinberg, S.F. 2000. Differential targeting of $\beta$-adrenergic receptor subtypes and adenylyl cyclase to cardiomyocyte caveolae. A mechanism to functionally regulate the cAMP signaling pathway. J. Biol. Chem. 275:41447-41457.

42. Kuschel, M., et al. 1999. $G_{i}$ protein-mediated functional compartmentalization of cardiac $\beta_{2}$-adrenergic signaling. J. Biol. Chem. 274:22048-22052.

43. Wright, S.C., Schellenberger, U., Ji, L., Wang, H., and Larrick, J.W. 1997. Calmodulin-dependent protein kinase II mediates signal transduction in apoptosis. FASEB J. 11:843-849.

44. Fladmark, K.E., et al. 2002. $\mathrm{Ca}^{2+} /$ calmodulin-dependent protein kinase II is required for microcystin-induced apoptosis. J. Biol. Chem. 277:2804-2811.

45. Xiao, R.P., et al. 1994. $\beta_{2}$-adrenergic receptor-stimulated increase in cAMP in rat heart cells is not coupled to changes in $\mathrm{Ca}^{2+}$ dynamics, contractility, or phospholamban phosphorylation. J. Biol. Chem. 269:19151-19156.

46. Daaka, Y., Luttrell, L.M., and Lefkowitz, R.J. 1997. Switching of the coupling of the $\beta_{2}$-adrenergic receptor to different $G$ proteins by protein kinase A. Nature. 390:88-91.

47. Yatani, A., and Brown, A.M. 1989. Rapid $\beta$-adrenergic modulation of cardiac calcium channel currents by a fast $G$ protein pathway. Science. 245:71-74.

48. Lader, A.S., et al. 1998. Cardiac $\mathrm{G}_{\mathrm{s} \alpha}$ overexpression enhances L-type calcium channels through an adenylyl cyclase independent pathway. Proc. Natl. Acad. Sci. U. S. A. 95:9669-9674.

49. Bristow, M.R. 2000. $\beta$-adrenergic receptor blockade in chronic heart failure. Circulation. 101:558-569.

50. Port, J.D., and Bristow, M.R. 2001. Altered $\beta$-adrenergic receptor gene regulation and signaling in chronic heart failure. J. Mol. Cell. Cardiol. 33:887-905. 